\title{
Espirómetro Electrónico Portátil con Visualización en Dispositivo Móvil
}

\author{
Portable Electronic Spirometer with Visual Interface on a Mobile Device
}

\author{
R. A. Díaz-Suarez iD ; V. A. Pabón-Castillo iD ; S. F. Vera-Medina iD , A. F. Gómez -Chacón iD
}

\begin{abstract}
Chronic respiratory diseases, such as COPD and Asthma, are responsible for millions of deaths in the world, and their prevalence has increased mainly due to the world population aging, exposure to tobacco smoke, and high environmental pollution. High mortality rates arise because these diseases are usually underdiagnosed or poorly diagnosed, and that disease treatment is not followed-up properly by a medical specialist. In this research work, we present the development of a portable, low cost spirometer that allows the visualization, analysis and storage, in an Android-based mobile device, of the air flow records obtained during lung capacity tests. Therefore, the developed prototype facilitates the diagnosis and monitoring of respiratory diseases.
\end{abstract}

Index Terms - Android, COPD, Spirometer, Respiratory Flux, Biomedical Signal Processing.

Resumen - Las enfermedades respiratorias crónicas, tales como el EPOC y el Asma, son responsables de millones de muertes en el mundo, y su prevalencia se ha incrementado principalmente debido al envejecimiento de la población mundial, la exposición al humo de tabaco, y la alta contaminación ambiental. Estas altas tasas de mortalidad se deben a que las enfermedades respiratorias son usualmente mal diagnosticadas o subdiagnosticadas, y que usualmente se carece de un adecuado seguimiento del tratamiento de la enfermedad por un médico experto. En este trabajo de investigación, se presenta el desarrollo de un espirómetro portable, de bajo costo, y que permite visualizar, analizar y almacenar, en un dispositivo móvil Android, los registros de flujo de aire obtenidos durante pruebas de capacidad pulmonar. Por lo tanto, el prototipo realizado permite facilitar el diagnóstico y seguimiento de enfermedades respiratorias.

Palabras claves- Android, EPOC, Espirómetro, Flujo Respiratorio, Procesamiento de Señales Biomédicas.

Este manuscrito fue enviado el 4 de abril del 2018 y aceptado 15 de marzo, de 2019. Este Proyecto fue financiado por la Universidad Manuela Beltrán.

R. A. Díaz-Suarez. Es docente del programa de ingeniería Biomédica de la Universidad Manuela Beltrán, Director del grupo SISTECBIO, Bucaramanga Santander Colombia (correo andres.diaz@ docentes.umb.edu.co).

V. A. Pabón-Castillo. Es ingeniero biomédico de la Universidad Manuela Beltrán. Bucaramanga, Santander Colombia (correo victor.pabon@academia.umb.edu.co)

\section{INTRODUCTION}

$\mathrm{L}$ AS enfermedades pulmonares y respiratorias son de las

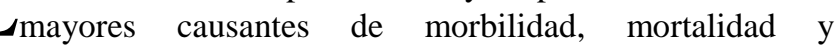
discapacidad en el mundo [1]. Entre ellas, la Enfermedad Pulmonar Obstructiva Crónica (EPOC) es la tercera causa de mortalidad en el mundo, mientras que el Asma es considerada la enfermedad no infecciosa más común en niños [2].

Uno de los principales retos para mejorar el diagnóstico y tratamiento efectivo de enfermedades respiratorias es la dificultad de acceso a dispositivos de diagnóstico [3]. Según la Organización Mundial de la Salud (OMS), en 2004 se estimó que 299 millones de personas sufrían de enfermedades respiratorias crónicas sin que estas llegasen a ser diagnosticadas [4]. La principal técnica de diagnóstico de enfermedades respiratorias es la espirometría, la cual determina y cuantifica la capacidad respiratoria de los pulmones, estimando el volumen del aire inhalado y exhalado durante una prueba de respiración forzada.

El avance de la tecnología médica, específicamente de la telemedicina, ha permitido desarrollar equipos electrónicos enfocados a la prestación de servicios médicos a distancia. Actualmente existe un interés creciente en desarrollar equipos de diagnóstico, de enfermedades respiratorias, sencillos, portables y de bajo costo. Usando equipos móviles, se cambia el paradigma en el monitoreo y diagnóstico de enfermedades respiratorias, ya que el mismo usuario tendrá la capacidad de monitorear su propia condición de salud, y el dispositivo le proporcionará información médica precisa de manera inmediata. Un beneficio adicional es que estos dispositivos permiten almacenar las mediciones realizadas y enviarlas de

S. F. Vera-Medina. Es ingeniero biomédico de la Universidad Manuela Beltrán. Bucaramanga, Santander Colombia (correo sebastian.vera@academia.umb.edu.co)

A. F. Gómez -Chacón. Es docente de la Universidad Manuela Beltran. Bucaramanga, Santander Colombia (correo: andres.gomez@docentes.umb.edu.co) 
forma simple a un médico especialista, quien no requiere estar presente en el sitio de la prueba.

Existen diversos modelos funcionales que permiten realizar la espirometría. En [2], [5], se propone una aplicación para realizar espirometría a partir de la señal de sonido captada por el micrófono integrado de un celular. En [1], se propone un sistema de análisis de la tos para identificar el tipo de enfermedad respiratoria, usando igualmente el micrófono integrado de un dispositivo móvil. Sin embargo, estas técnicas basadas en el uso de la señal de sonido son susceptibles al ruido del entorno en el que se encuentre el paciente. En [6], se propuso un espirómetro digital de bajo costo que transmite la señal captada hacia un dispositivo móvil con sistema operativo Android. Sin embargo, no se realiza una posterior prueba y comparación de la eficiencia y precisión de este dispositivo con respecto a un espirómetro estándar de uso médico. En [3], se propuso un prototipo de espirómetro que está directamente conectado a un smartphone. El sistema de sensado se basa en un espirómetro de turbina, el cual mide el flujo respiratorio a partir de las revoluciones de la turbina debido al paso del aire exhalado por el paciente. Este sistema tiene la desventaja de ser más complejo y de que sufre un desgaste con el tiempo, causando fricción en la turbina. Por tanto, su tiempo de vida útil puede ser reducido. Además, la operación misma de la turbina crea una carga al flujo de aire, lo que puede alterar las mediciones [7]. Por otra parte, sólo se realizó la comparación del sistema implementado con un sistema médico en base a un único paciente, lo que puede sesgar las conclusiones obtenidas en cuanto a la precisión del dispositivo.

En este trabajo se presenta el diseño y construcción de un sistema de espirometría portable de bajo costo usando como interfaz de usuario un dispositivo móvil con sistema operativo Android. El sistema de sensado de la capacidad respiratoria está basado en el espirómetro tipo Fleisch, el cual usa un sensor de presión, proporcionando mayor durabilidad del producto en comparación de los espirómetros de turbina. El hardware electrónico, y la interfaz gráfica de usuario (elaborada para el sistema operativo Android utilizando el IDE de processing) se describen en detalle. El costo estimado del dispositivo realizado es inferior a los 90USD. El prototipo elaborado permite el fácil diagnóstico y seguimiento de enfermedades respiratorias, mejorando así la calidad de vida de los usuarios.

A continuación, en la sección II se describen los fundamentos básicos de la técnica de espirometría. En la sección III se explica detalladamente el diseño del prototipo de espirómetro propuesto y el respectivo aplicativo móvil elaborado. La sección IV presenta los resultados obtenidos al comparar mediciones de capacidad pulmonar obtenidas con el prototipo propuesto respecto a un espirómetro comercial. Adicionalmente se presenta una descripción detallada del costo de materiales. Finalmente, en la sección $\mathrm{V}$ presenta las conclusiones de este trabajo.

\section{FUNDAMENTOS DE LA ESPIROMETRÍA}

La espirometría es la técnica más utilizada para medir la capacidad pulmonar de una persona. Esta técnica se basa en medir el volumen del aire inhalado y exhalado por los pulmones durante cierto período de tiempo. El procedimiento consiste en que el paciente realice una inhalación máxima y luego realice una exhalación del aire dentro del dispositivo espirómetro tan fuerte y rápido como sea posible. Durante ese proceso se mide el flujo de aire a lo largo del tiempo y el volumen total del aire exhalado.

\section{A. Neumotacógrafo}

Existen diferentes mecanismos para medir el flujo de aire, como el Neumotacógrafo de Turbina, el cual se basa en medir la cantidad de revoluciones de una turbina debido al flujo de aire que la impulsa, y el Neumotacógrafo de Resistencia Neumática Fleisch, el cual mide la diferencia de presión que se genera al pasar un flujo de aire laminar a través de una resistencia conocida [8]. Siendo este último ampliamente utilizado debido a su practicidad, bajo costo y la durabilidad [9].

El espirómetro desarrollado en este trabajo utiliza la técnica del Neumotacógrafo de Resistencia Neumática Fleisch. Donde se utiliza un arreglo de pequeños tubos capilares que crean un flujo pseudo-laminar, que como salida causan una presión proporcional lineal la cual se aplica a un transductor diferencial de presión. Los sensores y traductores de flujo que se utiliza normalmente en espirometría son los neumotacógrafos, son sensores diferenciales basados en la ley de Poiseuille que permite el cálculo del volumen a partir de la diferencia de la presión diferencial.

\section{B. Parámetros Principales de la Espirometría}

Como resultado de una prueba de espirometría se obtienen las curvas de Volumen de Aire vs. Tiempo, Flujo de Aire vs Tiempo, y Flujo de Aire vs. Volumen de Aire. En donde utilizando estas curvas se pueden extraer diversas métricas que son utilizadas para establecer diagnósticos. En la Fig. 1 y Fig. 2 se ilustran las curvas características obtenidas durante una prueba de espirometría. A continuación, se describen algunas de las principales métricas que se extraen de estas curvas:

- Flujo Espiratorio Máximo (PEF): Es el máximo flujo de aire durante la prueba.

- Volumen Espiratorio Forzado luego de 1 segundo (FEV1): Es el volumen total de aire exhalado durante un segundo. Este parámetro suele medirse para diferentes intervalos de tiempo (FEV2, FEV3, etc.)

- Capacidad Vital Forzada (FVC): Es el volumen total de aire exhalado durante la espirometría. 


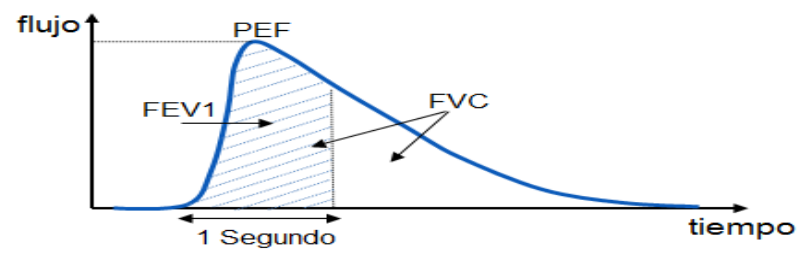

Fig1. Curva característica de flujo de aire vs tiempo como resultado de una espirometría.

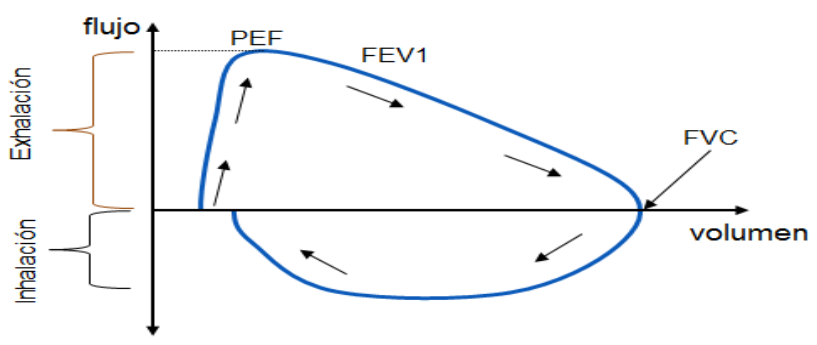

Fig. 2. Curva característica de flujo de aire vs volumen de aire como resultado de una espirometría.

Cuando un paciente presenta alguna enfermedad respiratoria, esto se ve reflejado en la forma de las curvas y en las respectivas métricas establecidas. Por ejemplo, si el paciente presenta una enfermedad obstructiva, la curva de volumen durante la inhalación tenderá a verse similar a la normal, sin embargo, el flujo de aire durante la exhalación y el volumen de aire exhalado serán menores, en comparación a una curva normal.

\section{PROTOTIPO DE ESPIRÓMETRo Portable PROPUESTO}

\section{A. Hardware}

El prototipo desarrollado consta de tres etapas: 1) Etapa de sensado, 2) Etapa de Adecuación de Señal, y 3) Etapa de Digitalización y Transmisión.

1)Etapa de Sensado. Para medir el flujo respiratorio se utilizó el sensor de presión diferencial MPX2010DP, el cual se muestra en la Fig. 3. Este es un transductor piezo-resistivo con compensación de temperatura y que permite medir un rango de presión de 0 a $10 \mathrm{kPa}$. El sensor genera una salida de tensión lineal y proporcional a la presión diferencial aplicada en las boquillas de entrada.

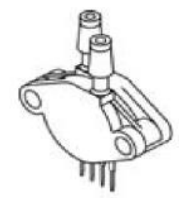

Fig. 3. Sensor de presión diferencial MPX2010DP [10].

Para captar el flujo de aire se diseñó un neumotacógrafo, el cual es un cilindro diseñado para generar una resistencia neumática en su interior, lo que genera un diferencial de presión que crece al aumentar el flujo de aire. La resistencia neumática se realiza mediante un sistema de mallas que causan una leve obstrucción al paso de flujo de aire, como se observa en la Fig. 4. Cuando se usa un mayor número de mallas, la resistencia neumática incrementa, por tanto, también lo hace el diferencial de presión generado. El diferencial de presión que se usa como entrada al sensor MPX2010DP se obtiene de medir la presión antes y después de las mallas.

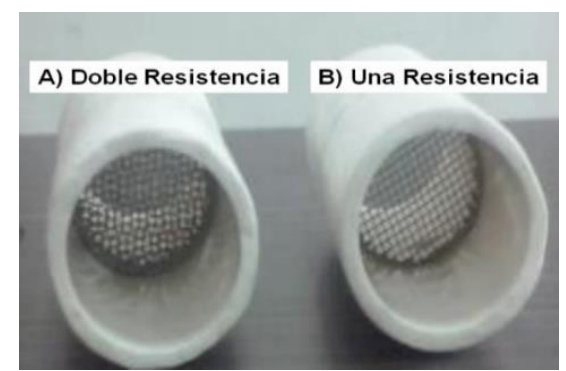

Fig. 4. Neumotacógrafo: La resistencia neumática se genera con las mallas en su interior.

2)Etapa de Acondicionamiento de Señal. Las etapas del hardware de la adecuación de señal se ilustran en la Fig. 5. El voltaje diferencial de salida del sensor de presión es captado por el amplificador de instrumentación AD620AN para realizar una pre-amplificación de la forma de onda. Este amplificador tiene un alto CMRR (100dB), lo cual permite reducir el ruido de modo común de la señal y así procesar una señal limpia en las etapas siguientes. La ganancia del pre-amplificador se fijó a 500.

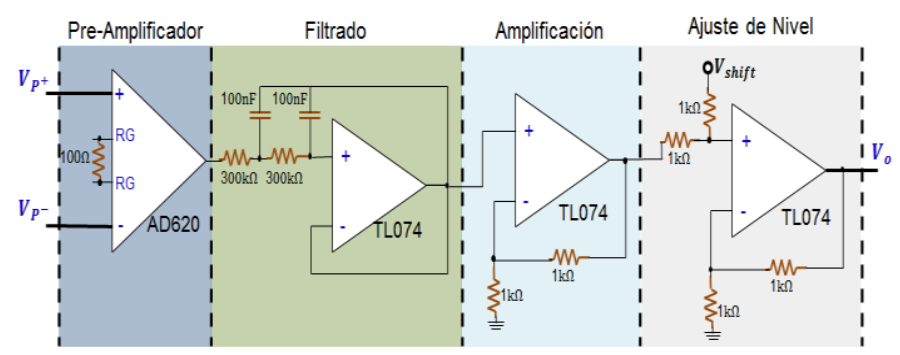

Fig. 5. Circuito de adecuación de señal

En la literatura se encuentran diferentes opiniones entre el estándar del rango de frecuencias a filtrar la señal de flujo respiratorio [11]. En [12], se analizó el espectro de la señal respiratoria durante pruebas de respiración forzada y se concluyó que el contenido armónico significativo de la señal se encuentra entre DC y $6.5 \mathrm{~Hz}$. Para filtrar la señal se implementó un filtro pasa-bajas de segundo orden. Para el prototipo, se fijó la frecuencia de corte del filtro a $5.3 \mathrm{~Hz}$. La señal filtrada y con bajo ruido de modo común es amplificada en un factor de 2 . Finalmente, se ajusta el nivel de la señal de salida para que esta no tome valores negativos durante la inhalación, y así la señal de flujo pueda ser adquirida correctamente por medio de un microcontrolador. El ajuste de nivel se realiza con un simple circuito sumador (ver Fig. 5) donde el voltaje de ajuste $V_{\text {shift }}$ es un voltaje de DC que define que tanto se va a desplazar la señal verticalmente. Basándose en diferentes pruebas del circuito para diferentes niveles de presión diferencial, se determinó que Vshift $=0.45 \mathrm{~V}$ es suficiente para garantizar que la señal de salida del circuito de adecuación esté entre 0 y $5 \mathrm{~V}$. 


\section{B. Digitalización y transmisión de la señal}

El microcontrolador utilizado para la adquisición de la señal de flujo respiratorio fue el ATMEGA328P, el cual se programó usando el IDE Arduino. La señal se adquiere por medio de un puerto configurado como canal conversor Analógico/Digital con una frecuencia de muestreo de $50 \mathrm{~Hz}$. La señal digitalizada es transmitida por medio del módulo de Bluetooth HC-06 a un celular con sistema operativo Android. El módulo de bluetooth tiene un alcance entre $5 \mathrm{~m}$ y $10 \mathrm{~m}$, lo que permite eliminar cableado innecesario y mejorar la comodidad del paciente durante la prueba de espirometría.

\section{Aplicativo Software para Android}

Se desarrolló un aplicativo software para Android usando el IDE Processing for Android. En la Fig. 6 se presenta el diagrama de flujo del aplicativo software desarrollado.

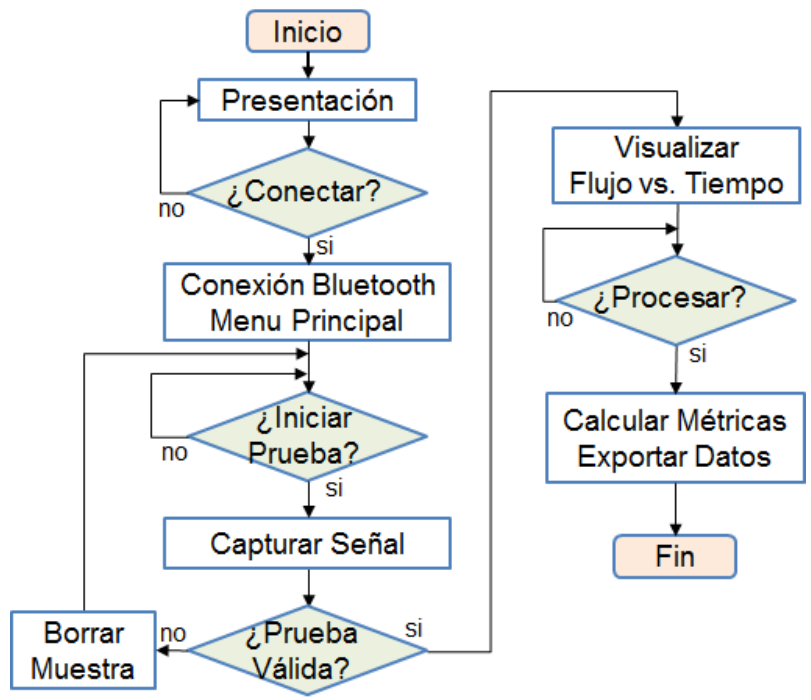

Fig. 6. Diagrama de flujo

Inicialmente se requiere que el usuario seleccione el dispositivo bluetooth correspondiente al espirómetro del cual se recibirán los datos de la señal de flujo respiratorio. Una vez la conexión bluetooth es establecida, se presenta el menú principal donde se le solicita al usuario que realice la prueba de espirometría. $\mathrm{Al}$ iniciar la prueba, se inicia la captura de la señal de flujo respiratorio por medio del microcontrolador. Si la prueba es satisfactoria, se grafica la curva de flujo vs tiempo. El usuario debe oprimir un botón con la opción de procesar la señal y graficar los datos de la prueba realizada.

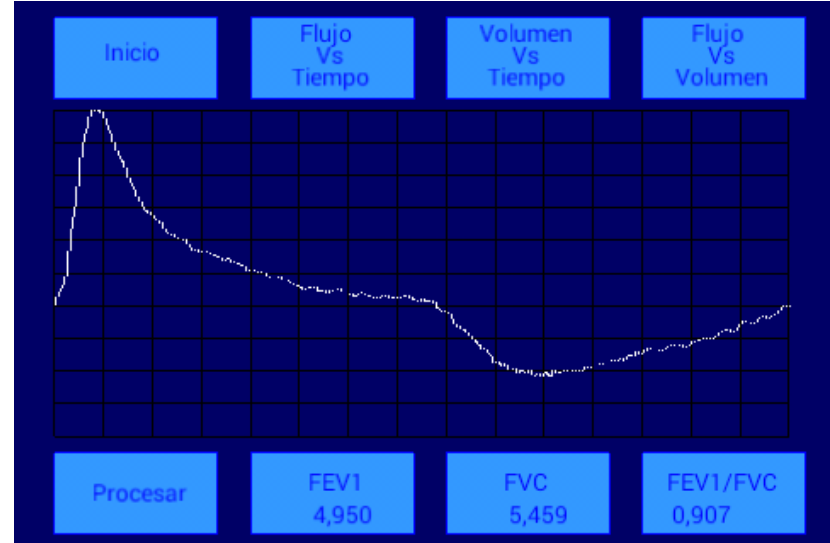

Fig. 7. Curva de flujo de aire vs tiempo visualizada en la aplicación móvil desarrollada.

En la Fig. 7 se muestra la interface visual de la aplicación móvil junto con la curva de flujo vs tiempo obtenida de la prueba de un usuario. Como puede observarse, se tiene la opción de visualizar las curvas de flujo vs tiempo, volumen vs tiempo y flujo vs volumen. Además, al procesar la señal de flujo respiratorio se calculan los parámetros de interés de la espirometría, tales como FEV1, FVC y FEV1/FVC. Cuando una prueba no es satisfactoria, los datos se borran y se pide repetir la prueba. Finalmente, la aplicación desarrollada genera un archivo texto con los datos obtenidos de la prueba de espirometría. Este archivo es almacenado en la memoria interna del dispositivo móvil para que luego pueda ser procesado utilizando otras herramientas de análisis, tales como MATLAB, o para que los resultados de la prueba puedan ser enviados a un médico experto para su posterior análisis.

\section{RESUltados Y VALIDACión DE FUNCIONAMIENTO}

El hardware final del prototipo implementado se presenta en la Fig 8. El PCB del circuito se realizó utilizando dos capas para facilitar el ruteado del circuito y minimizar la posible interferencia entre diferentes líneas de metal. Las dimensiones del PCB son de $7 \mathrm{~cm} \times 12 \mathrm{~cm}$. El PCB cuenta con cuatro puertos USB. Uno de ellos se usa para conectar el sensor de presión, y los otros tres se usan como fuentes de alimentación, las cuales pueden ser directa o por medio de una batería. Las zonas indicadas en la Fig. 8 se explican a continuación:

1. Etapa de filtrado

2. Etapa de Pre-amplificación.

3. Etapa de inversor de voltaje.

4. Puerto USB conexión cable alimentación.

5. Puerto USB conexión sensor de presión.

6. Puertos USB fuentes alternas de alimentación.

7. Conexión para el módulo bluetooth.

8. Micro controlador ATMEGA328. 


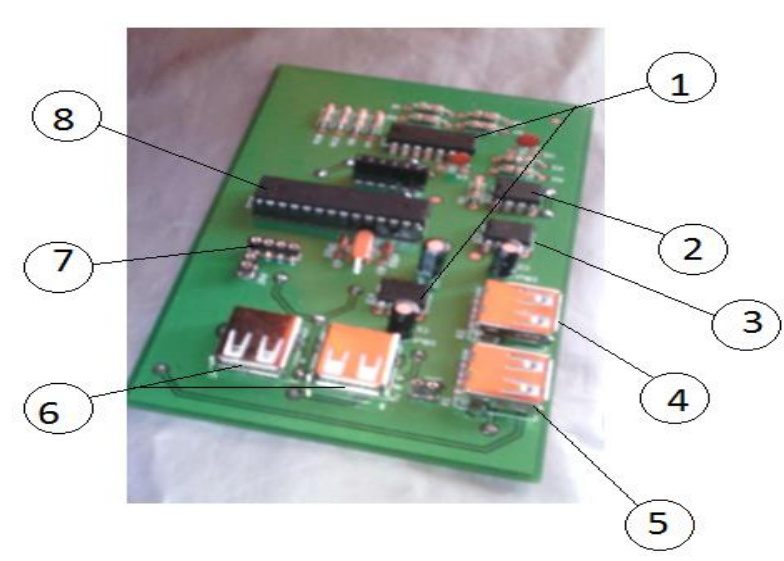

Fig. 8. Hardware final del prototipo.

El dispositivo final desarrollado se muestra en la Fig. 9. La carcasa que contiene el hardware electrónico y el mango del neumotacógrafo fueron diseñados en material acrílico.

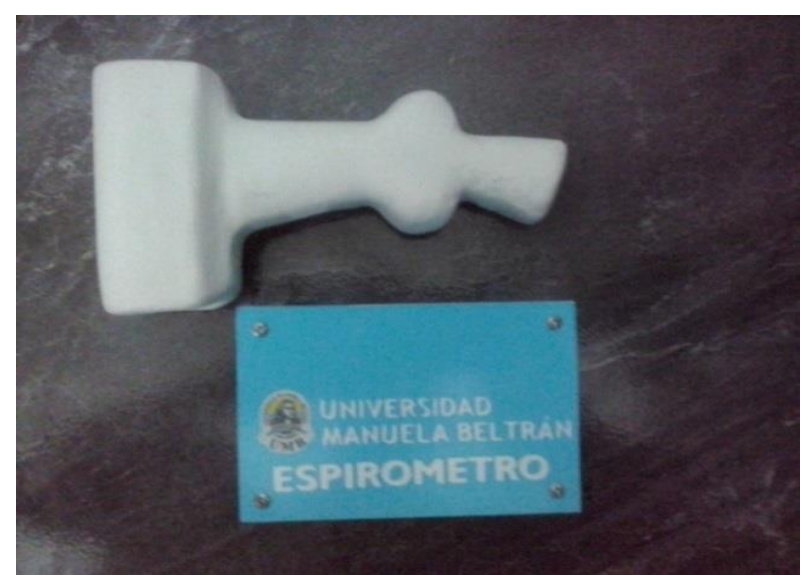

Fig. 9. Dispositivo espirómetro final

Para probar el prototipo desarrollado y verificar su correcto funcionamiento, se realizaron pruebas en 9 voluntarios obteniendo mediciones simultáneas con el prototipo realizado y con un espirómetro de mesa comercial de la marca MicroLab ${ }^{\mathrm{TM}}$, el cual se usa como referencia. Para realizar esta prueba, se unieron las boquillas de los mangos de los dos espirómetros de tal forma que ambos reciben el mismo flujo.

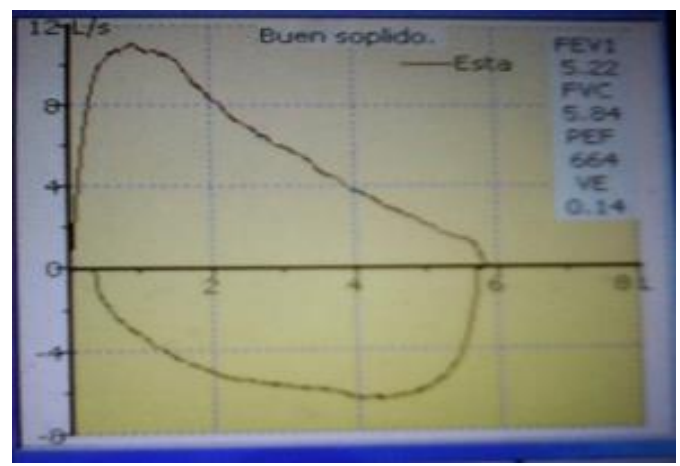

a) Espirómetro de referencia

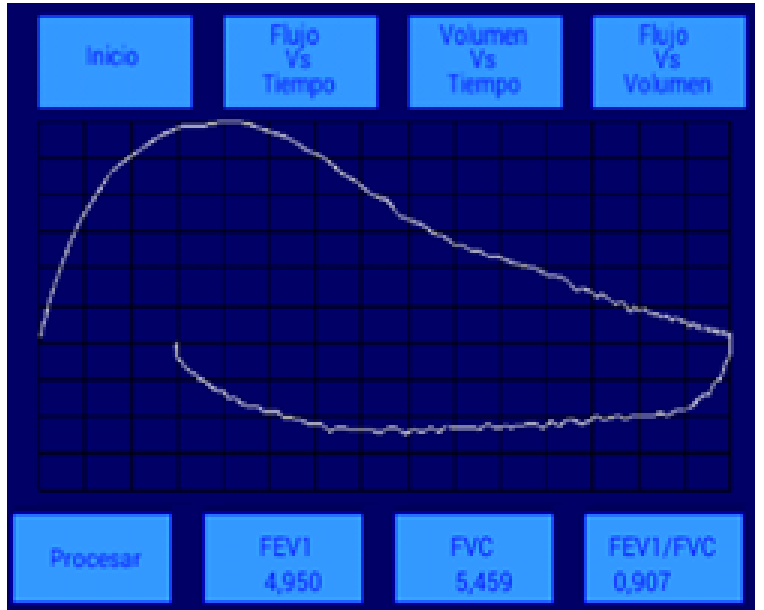

b) Espirómetro desarrollado.

Fig. 10. Ejemplo comparación de las medidas equipo vs espirómetro elaborado.

En la Fig. 10 se muestra una comparación de la curva de flujo vs tiempo obtenida con el espirómetro de referencia (Fig. 10a) y con el espirómetro desarrollado (Fig. 10b). Como puede observarse, la forma de la curva y los valores de las métricas de FEV1 y FVC son bastante similar en ambos equipos.

Las Fig. 11 y Fig. 12 presentan una comparación de los datos de espirometría (PEF y FVC) obtenidos para los 9 voluntarios mediante el espirómetro de referencia y mediante el prototipo de espirómetro propuesto. Como se puede observar, los valores obtenidos con el prototipo son bastante cercanos a los obtenidos con el dispositivo comercial. En promedio, el error en la medición de PEF y el error en la medición de FVC es de $2.75 \%$ y $6.37 \%$, respectivamente. Estos resultados demuestran que el prototipo diseñado tiene una buena precisión y puede ser utilizado para un diagnóstico oportuno de enfermedades respiratorias.

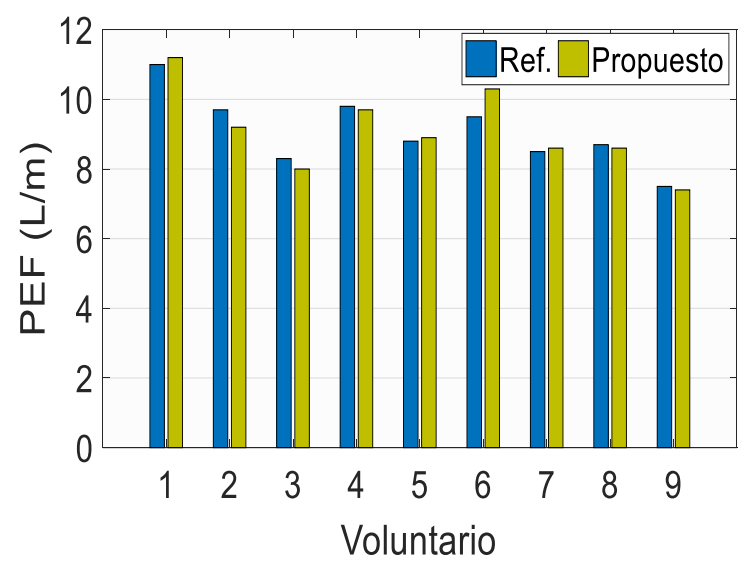

Fig. 11. Comparación de mediciones obtenidas de flujo espiratorio máximo (PEF). 


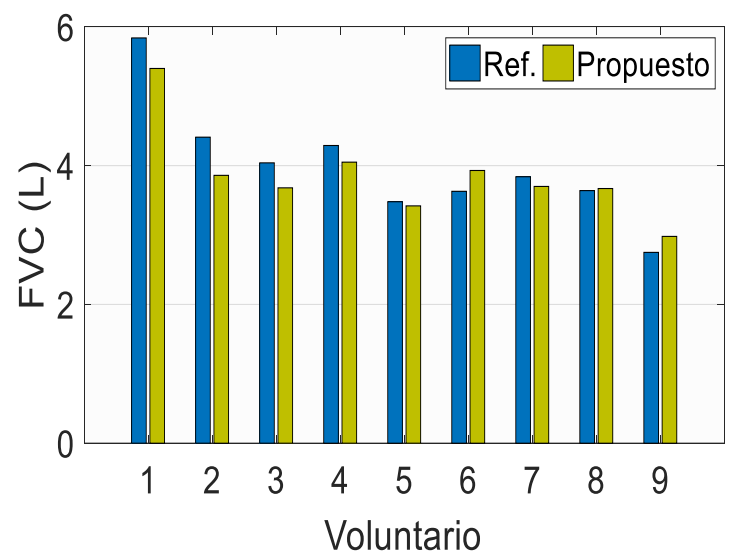

Fig. 12. Comparación de mediciones obtenidas de capacidad vital forzada (FVC)

Una cotización de los materiales empleados para desarrollar el prototipo se muestra en la Tabla I. Aunque algunos elementos como el microcontrolador y el módulo de Bluetooth tienen un valor definido en el mercado, otros elementos como la carcasa y el diseño de la PCB pueden ser reducidos significativamente. Aún más, el diseño de la PCB no afecta directamente el costo de fabricación en masa. El costo total del espirómetro desarrollado fue de $\$ 250.000$ COP ( 90USD). Es de resaltar que este valor está muy por debajo del costo de otros espirómetros comerciales, el cual oscila entre $\$ 2^{`} 000.0000$ y $\$ 8^{\prime} 000.000 \mathrm{COP}$.

\section{TABLA I. COSTO DE MATERIALES}

\begin{tabular}{lc}
\hline \hline \multicolumn{1}{c}{ Elemento } & Precio (COP) \\
\hline Microcontrolador ATMega328 & $\$ 15.000$ \\
Módulo Bluetooth & $\$ 50.000$ \\
Elementos Electrónicos & $\$ 20.000$ \\
(Resistencias, amplificadores, etc.) & \\
Sensor Presión Diferencial & $\$ 20.000$ \\
Batería Recargable & $\$ 15.000$ \\
Carcasa & $\$ 80.000$ \\
Diseño PCB* & $\$ 50.000$ \\
Total & $\mathbf{2 5 0 . 0 0 0}$ \\
\hline \hline
\end{tabular}

* El costo del diseño del a PCB no afecta la fabricación en masa.

\section{CONCLUSIONES}

Se ha presentado el desarrollo de un prototipo de espirómetro portable con visualización e interfaz de usuario por medio de un dispositivo móvil con sistema operativo Android. Para adquirir la señal de flujo respiratorio se ha usado un neumotacógrafo tipo Fleisch, el cual usa un sensor piezoeléctrico de presión diferencial. Este método de sensado es más robusto que el clásico uso de turbinas. El prototipo envía los resultados de la prueba a un celular por medio del módulo bluetooth. La aplicación desarrollada extrae diferentes métricas de interés de la prueba de espirometría como el FVC, PEF, etc. Los resultados son almacenados en el dispositivo movil en un archivo texto, el cual puede ser fácilmente compartido a un médico experto para que este visualice los resultados obtenidos y pueda dar un diagnóstico oficial. Se comparó las mediciones realizadas con el prototipo diseñado y con un espirómetro de mesa comercial de la MicroLab ${ }^{\mathrm{TM}}$, demostrándose que el prototipo propuesto realiza mediciones muy cercanas al espirómetro comercial.

\section{REFERENCIAS}

[1] D. B. C. R. K. a. R. R. F. C. Infante, "Classification of voluntary coughs applied to the screening of respiratory disease", de 2017 39th Annual International Conference of the IEEE Engineering in Medicine and Biology Society (EMBC), Seogwipo, 2017. DOI: 10.1109/EMBC.2017.8037098

[2] A. S. F. A. a. H. M. F. Zubaydi, "MobSpiro: Mobile based spirometry for detecting COPD",de 2017 IEEE 7th Annual Computing and Communication Workshop and Conference $(C C W C)$, Las Vegas, NV, USA, 2017. DOI: 10.1109/CCWC.2017.7868345

[3] S. S. a. S. M. S. Bagchi, "Development and Characterization of a Wireless Mouse-Based Spirometer", IEEE Sensors Journal, vol. 17, no 7, pp. 2065-2073, 2017. DOI: 10.1109/JSEN.2017.2664889

[4] W. [Online], "About chronic respiratory diseases,» Available: http://www.who.int/respiratory/about_topic/en/.

[5] E. C. L. e. al, "SpiroSmart: using a microphone to measure lung function on a mobile phone", de 2012 ACM Conference on Ubiquitous Computing (UbiComp '12), New York, NY, USA, 2012. DOI: $10.1145 / 2370216.2370261$

[6] C. A. a. G. D. C. C. W. Carspecken, "TeleSpiro: A low-cost mobile spirometer for resource-limited settings", de 2013 IEEE Point-ofCare Healthcare Technologies (PHT), Bangalore, India, 2013. DOI: 10.1109/PHT.2013.6461305

[7] I. Hariadi, "Sensitivity analysis of silicon MEMS Thermal Flow Sensor for spirometer application", de 2nd International Conference on Instrumentation, Communications, Information Technology, and Biomedical Engineering (ICICI-BME), 2011 , Bandung, Indonesia, 2011. DOI: 10.1109/ICICIBME.2011.6108619

[8] F. G.-R. e. al, "Espirometría", Archivos de Bronconeumología, vol. 49, nº 9, pp. 388-401, 2013.

[9] *. C. J. D.-M. N. Alejos-Palomares R., "Digital Spirometer with LabView Interface", de 18th International Conference on Electronics, Communications and Computers, Puebla, México, 2008. DOI: 10.1109/CONIELECOMP.2008.31

[10] N. F. Semiconductor, "MPX2010 Datasheet", [En línea]. Available: sheet/MPX2010.pdf.

[11] Y. S. M. E. a. I. M. V. Lopata, "On the spirometry and spirometers standardization", de 2013 IEEE XXXIII International Scientific Conference Electronics and Nanotechnology (ELNANO), Kiev, 2013. DOI: 10.1109/ELNANO.2013.6552028

[12] G. C. W. M. P. K. Lemen RJ, "Frequency spectra of flow and volume events for forced vital capacity", J Appl Physiol Respir Environ Exerc, vol. 53, pp. 977-984, October 1982. DOI: 10.1152/jappl.1982.53.4.977

[13] D. d. P. y. Prevención, "ENFERMEDAD PULMONAR OBSTRUCTIVA CRÓNICA - EPOC”, Bogotá, 2013.

[14] W. [Online], "WHO | The top 10 causes of death", Available: http://www.who.int/mediacentre/factsheets/fs310/en/, 2017.

[15] A. C. e. al, "Prevalence of COPD in Five Colombian Cities Situated at Low, Medium, and High Altitude (PREPOCOL Study)", CHEST, vol. 133, n ${ }^{\circ}$ 2, pp. 343 - 349, 2008. DOI: $10.1378 /$ chest.07-1361

[16] L. e. a. GOCHICOA-RANGEL, "Oscilometría de impulso: Recomendaciones y procedimiento", Neumología y cirugía de tórax [revista en la Internet], vol. 73, $\mathrm{n}^{\mathrm{o}}$ 2, pp. 138-149, 2014.

[17] J. V. a. A. Johnson, "An Effortless Noninvasive Respiratory Diagnostic Device", de 2016 32nd Southern Biomedical Engineering Conference (SBEC), Shreveport,LA, 2016. DOI: 10.1109/SBEC.2016.96 
[18] O. A. B. Rodríguez, "Diseño e implementación de un espirómetro", Tekhnê, vol. 10, n 2, pp. 5-14, 2013.

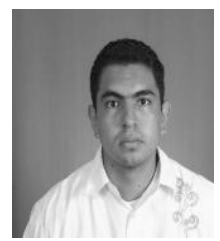

Ricardo Andrés Díaz Suarez, nació en el Socorro, Santander, Colombia en 1983. El recibió el grado de ingeniero electrónico y magister en ingeniería electrónica de la Universidad Industrial de Santander en el año 2007 y 2011 respectivamente. Es docente del programa de Ingeniería Biomédica en la Universidad Manuela Beltrán desde el año 2012. En la actualidad sus líneas de investigación están enfocadas al desarrollo de equipo médico y en el área de las comunicaciones aplicadas, está categorizado como investigador asociado por Colciencias.

ORCID: http://orcid.org/0000-0003-1590-6860

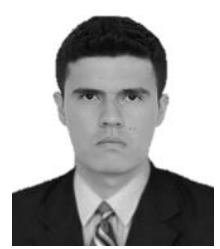

Victor Alfonso Pabon Castillo, nació en Bucaramanga, Santander, Colombia en 1992. El recibió el grado de ingeniero Biomédico de la Universidad Manuela Beltrán en Bucaramanga en el año 2017. En la actualidad se encuentra laborando en una empresa enfocada al desarrollado de equipos médicos para la adquisición, procesamiento y visualización de señales fisiológicas, destacando entre estos dispositivos un espirómetro portátil y un tensiómetro digital. ORCID: http://orcid.org/00000003-0863-1504

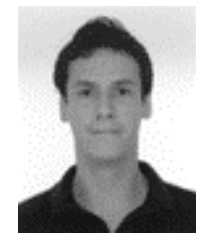

Sebastian Fernando Vera Medina, nació en Duitama, Boyacá, Colombia en el año de 1994. En el año 2012 decide estudiar ingeniería biomédica debido a su interés en el desarrollo de tecnología medica para mejorar la atención en salud. En el año 2017 recibe el título de ingeniero biomédico en la ciudad de Bucaramanga y donde culmina su proyecto de investigación en la creación de un espirómetro portable. Actualmente se encuentra trabajando en procesos para el mejoramiento de la salud en Colombia en personas de bajos recursos económicos. ORCID: http://orcid.org/0000-0001-8082-7750

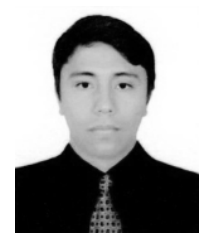

Andrés Felipe Gómez Chacón, es Ingeniero Electrónico (2011) de la Universidad Industrial de Santander (UIS), Colombia, y obtuvo los grados de Maestría y Doctorado en Ciencias de la Electrónica por parte del Instituto Nacional de Astrofísicca, Óptica y Electrónica (INAOE), México, en 2013 y 2017, respectivamente. Sus actuales intereses de investigación incluyen diseño, prueba y confiabilidad de de circuitos integrados digitales y diseño de circuitos electrónicos para aplicaciones de la industria médica y automotriz.

ORCID: http://orcid.org/0000-0002-4957-0448 